\title{
Assessment of Sleep Disturbances and Exhaustion in Mothers of Children With Atopic Dermatitis
}

Faustine D. Ramirez, BA; Shelley Chen, BS; Sinéad M. Langan, FRCP, PhD; Aric A. Prather, PhD; Charles E. McCulloch, PhD; Sharon A. Kidd, MPH, PhD; Michael D. Cabana, MD, MPH; Mary-Margaret Chren, MD; Katrina Abuabara, MD, MA, MSCE

IMPORTANCE The well-being and development of children is strongly influenced by parents' physical and psychosocial health. Data from small, clinic-based studies suggest that sleep loss may be common in parents of children with atopic dermatitis (AD), but longitudinal population-based studies are lacking.

OBJECTIVES To compare sleep disturbances over time between mothers of children with and without $A D$ and to determine whether these disturbances are associated with the child's disease severity and the child's sleep disturbances.

DESIGN, SETTING, AND PARTICIPANTS In the ongoing Avon Longitudinal Study of Parents and Children, all pregnant women residing in Avon, United Kingdom, with an expected delivery date between April 1, 1991, and December 31, 1992, were recruited. Analyses for this study, a secondary analysis of this cohort, were performed from September 2017 to September 2018. Mother-child pairs were followed up with a time-varying measure of child AD activity and severity and self-reported maternal sleep measures repeated at multiple time points between child ages 6 months and 11 years.

MAIN OUTCOMES AND MEASURES Time-varying binary measures of maternal sleep duration ( $<6$ vs $\geq 6$ hours per night), difficulty falling asleep, early morning awakening, subjectively insufficient sleep, and daytime exhaustion.

RESULTS The study followed up 13988 mother-child pairs from birth for a median duration of 11 (interquartile range, 7-11) years. Among the cohort, 11585 of 13972 mothers (82.9\%) were aged 21 to 34 years and 12001 of 12324 (97.4\%) were of white race/ethnicity; 7220 of 13978 children (51.7\%) were male. Sleep duration (adjusted odds ratio [AOR], 1.09; 95\% Cl, 0.90-1.32) and early morning awakenings (AOR, 1.16; 95\% Cl, 0.93-1.46) were similar between mothers of children with and without $A D$. In contrast, mothers of children with $A D$ were more likely to report difficulty falling asleep (AOR, 1.36; 95\% Cl, 1.01-1.83), subjectively insufficient sleep (AOR, 1.43; 95\% Cl, 1.24-1.66), and daytime exhaustion (AOR, 1.41; 95\% Cl, 1.12-1.78) independent of the child's comorbid asthma and/or allergic rhinitis. For all measures, worse child AD severity was associated with worse maternal sleep outcomes. The magnitude and significance of the associations were largely unchanged after adjustment for child sleep disturbances.

CONCLUSIONS AND RELEVANCE Mothers of children with AD reported difficulty falling asleep, subjectively insufficient sleep, and daytime exhaustion throughout the first 11 years of childhood. However, child sleep disturbances did not fully explain maternal sleep disturbances, and future research should investigate other mechanisms. In caring for children with $A D$, clinicians should consider maternal sleep disturbances and caregiver fatigue. 
T he health, well-being, and development of children is strongly influenced by the physical and psychosocial health of parents. ${ }^{1,2}$ Parents of children with chronic illness, in particular, are susceptible to poor sleep, and impaired sleep has been associated with increased risks of cancer and cardiovascular disease, ${ }^{3}$ infectious illness, ${ }^{4}$ obesity, ${ }^{5}$ and premature mortality. ${ }^{6}$ Previous studies have found major sleep impairments among parents of children with ventilator dependency and cystic fibrosis, ${ }^{7,8}$ but few studies to date have examined sleep patterns among parents of children with more common chronic illnesses.

Atopic dermatitis (AD) is one of the most common chronic childhood conditions, affecting up to 1 in 5 children in developed countries. ${ }^{9}$ Atopic dermatitis is characteristically itchy, and the symptom is often reported to be more severe at night, ${ }^{10,11}$ causing nighttime awakenings secondary to itching and scratching. ${ }^{12,13}$ To our knowledge, few studies have directly examined the association between parental sleep disturbances and severity of child $\mathrm{AD}$ and accounted for child sleep disturbances. Existing research is limited and has focused on snapshots of small, clinic-based populations that are likely to have more severe disease than the general population. ${ }^{12-15} \mathrm{Be}-$ cause the severity and activity of AD can vary throughout childhood, longitudinal studies can provide better insight into how variations in the child's disease activity and severity over time affect parental sleep.

We aimed to determine whether mothers of children with active $\mathrm{AD}$ have impaired sleep during the first 11 years of childhood and whether these sleep disturbances are associated with the child's disease severity and the child's sleep disturbances.

\section{Methods}

\section{Participants}

Data for this study come from the ongoing Avon Longitudinal Study of Parents and Children (ALSPAC), a longitudinal, population-based birth cohort in the United Kingdom. ${ }^{16,17}$ All pregnant women residing in Avon, United Kingdom, with an expected delivery date between April 1, 1991, and December 31, 1992, were recruited. The ALSPAC study enrolled a total of 14541 pregnancies, which resulted in 14062 live births, of which 13988 infants were alive at 1 year of age. For our analyses, we used data from 11649 mother-child pairs who had data on $\mathrm{AD}$ from at least 1 survey and sleep outcomes from at least 1 survey through age 11 years $(83.3 \%$ of children who remained alive at 1 year of age). The study website contains details of all the data available through a fully searchable data dictionary and variable search tool. ${ }^{18}$ Data collection began in 1990 through 1992, and analyses for this study, a secondary analysis of this cohort, were performed from September 2017 through September 2018. Ethical approval for the study was obtained from the ALSPAC Ethics and Law Committee and the Local Research Ethics Committees. The University of California, San Francisco declared the study exempt from the need for institutional review board approval because all data obtained by investigators were fully deidentified. Participants pro-

\section{Key Points}

Question Do mothers of children with atopic dermatitis experience sleep disturbances, and to what extent are these explained by child sleep disturbances?

Findings In this cohort study of 11649 mother-child pairs, sleep disturbances were common among mothers of children with atopic dermatitis followed up from birth through age 11 years. Having a child with atopic dermatitis was significantly associated with impaired maternal sleep quality, subjectively insufficient sleep, and increased daytime exhaustion; however, child sleep disturbances did not fully explain maternal sleep disturbances.

Meaning Clinicians caring for children with atopic dermatitis should screen for maternal sleep disturbances and caregiver fatigue.

vided written informed consent at enrollment into the original cohort.

\section{Exposure}

The primary exposure was a time-varying measure of active $\mathrm{AD}$ in the child. Mothers were asked a standardized question about flexural dermatitis at 10 time points between child ages 6 months and 11 years $(6,18,30,42,57,69,81,103,128$, and 140 months): "Has your child had an itchy, dry skin rash in the joints and creases of his body (eg, behind the knees, elbows, under the arms) in the past year?” This question is comparable to that used in the large International Study of Asthma and Allergies in Childhood (ISAAC). ${ }^{19}$ Children were categorized as having active $\mathrm{AD}$ if their mother reported flexural dermatitis at least twice, up to and including the time point being considered. ${ }^{20-22}$ Disease severity was assessed at each time point by asking mothers to categorize their child's flexural dermatitis over the past year as no problem, mild, moderate, or severe.

\section{Maternal Sleep Outcomes}

Five maternal sleep outcomes were measured at various time points throughout childhood (Table1). Sleep duration was measured by a standardized categorical question repeated at 5 time points: "How many hours of sleep do you get altogether now during an average night?" (Answer choices: 1-3 hours, 4-5 hours, 6-7 hours, and $>7$ hours). This variable was modeled as a dichotomous outcome-fewer than 6 hours per night compared with 6 or more hours per night-because the National Sleep Foundation recommends 7 to 9 hours of sleep for adults aged 26 to 64 years. ${ }^{23}$ Difficulty falling asleep was assessed at 3 time points by the question, "Can you go to sleep alright?" (Answer choices: never/not very often vs very often/often), and early morning awakening was assessed at the same 3 time points by the question, "Do you wake unusually early in the morning even when you haven't been woken by your child or family?" (Answer choices: very often/often vs never/not very often). Subjectively insufficient sleep was examined at 4 time points by the question, "Do you feel that you are getting enough sleep?” (Answer choices: yes/no). Finally, daytime exhaustion was measured at 7 time points by the question, "In the past month, how often have you felt exhausted?" (Answer choices: almost always vs sometimes/not at all.) 


\begin{tabular}{lllllllll}
\hline Table 1. Maternal Sleep Outcome Measurements by Child Age \\
\begin{tabular}{lllllllll}
\hline \\
Child Age, mo $^{\mathrm{a}}$
\end{tabular} \\
\cline { 2 - 10 } Outcome & 21 & 33 & 47 & 61 & 73 & 85 & 110 & 134 \\
\hline Sleep duration & $\mathrm{x}$ & $\mathrm{x}$ & $\mathrm{x}$ & $\mathrm{x}$ & & $\mathrm{x}$ & & $\mathrm{x}$ \\
\hline Early morning awakening & $\mathrm{x}$ & $\mathrm{x}$ & & $\mathrm{x}$ & & & & \\
\hline Difficulty falling asleep & $\mathrm{x}$ & $\mathrm{x}$ & & $\mathrm{x}$ & & & & \\
\hline Subjectively insufficient sleep & $\mathrm{x}$ & $\mathrm{x}$ & & $\mathrm{x}$ & & $\mathrm{x}$ & & \\
\hline Daytime exhaustion & $\mathrm{x}$ & $\mathrm{x}$ & $\mathrm{x}$ & $\mathrm{x}$ & $\mathrm{x}$ & & $\mathrm{x}$ & $\mathrm{x}$ \\
\hline
\end{tabular}

an $\mathrm{x}$ indicates that the outcome was reported at the time point.

\section{Additional Covariates}

Several potential confounders, mediators, and effect modifiers were identified from earlier literature and incorporated into a directed acyclic graph that was used to guide our modeling strategy (eFigure in the Supplement). These factors included child and mother demographic characteristics (child sex, child age, mother's race/ethnicity, and maternal age at delivery), indicators of socioeconomic status, household smoking exposure, presence of comorbid atopic diseases in the child (asthma or allergic rhinitis), maternal sleep problems during pregnancy, maternal history of any atopic condition (AD, asthma, or allergic rhinitis), and child sleep disturbances. Socioeconomic status was measured using prenatal questionnaires collected from parents at study enrollment, including the mother's highest educational attainment, social class based on occupation (highest of either parent), household crowding index (number of people living in the household divided by the number of rooms in the house), and a financial difficulties score assessing the mother's self-reported difficulty to afford food, clothing, heating, rent or mortgage, and items necessary to care for her baby. Maternal sleep problems during pregnancy were assessed by prenatal questionnaire and based on questions about difficulty falling asleep at 18 and 32 weeks' gestation.

Time-varying covariates included child comorbid atopic disease, household smoking exposure, and child sleep disturbances. A child was determined to have comorbid atopic disease at each time point examined if his or her mother reported asthma symptoms, allergic rhinitis symptoms, or both at that time, on questions similar to those used in the ISAAC studies. ${ }^{19}$ Models also included a measure of household smoking exposure, which was assessed by maternal questionnaire about the number of smokers living in the household at multiple time points throughout childhood. Finally, child sleep disturbances were measured by a standardized question about the frequency of nighttime awakenings (dichotomized at $\geq 1$ awakening per night) at 6 time points in early childhood (30, 42, 57, 69,81 , and 115 months). Child nighttime awakenings have been shown to be a valid measure of $\mathrm{AD}$-associated child sleep disturbances throughout childhood. ${ }^{24}$

\section{Missing Data}

As has been described in detail elsewhere, there are both intermittent missing data and attrition (ie, loss to follow-up) in the ALSPAC cohort. ${ }^{16}$ Multiple imputation was used to impute missing exposure, outcome, and covariate data. Iterative chained equations were used because most variables in our models did not follow a normal distribution. Fifty im- puted data sets were generated and used to repeat primary analyses.

\section{Statistical Analysis}

Cross-sectional logistic regression analyses were performed to compare maternal sleep outcomes between mothers of children with and without $\mathrm{AD}$ at each time point. Longitudinal analyses with repeated measures of the exposure, outcome, and time-varying covariates were then performed using mixedeffects models with random slopes and intercepts for each individual to estimate the participant-specific odds ratios (ORs) for each of the maternal sleep outcomes. The minimally sufficient adjustment set was determined using our directed acyclic graph (eFigure in the Supplement), and these factors were included in multivariable models. Multivariable model 1 represents the fully adjusted model used to calculate the total association of child AD with maternal sleep, and model 2 was additionally adjusted for child sleep disturbances at each time point to estimate the direct association of child $\mathrm{AD}$ with maternal sleep, independent of child sleep disturbances. Interactions between child AD and child comorbid atopic disease (asthma or allergic rhinitis), child age, child sex, and maternal educational attainment were tested. All statistical analyses were performed using Stata, version 14.2 (StataCorp). Statistical significance was set at $P<.05$.

\section{Results}

The study sample was composed of 13988 mother-child pairs. Among the children, 7220 of 13978 (51.7\%) were male. Of the mothers, 12001 of 12324 (97.4\%) were of white race/ ethnicity, and 11585 of 13972 (82.9\%) were aged 21 to 34 years. The sample was followed for a median duration of 11 years (interquartile range, 7-11 years). Mothers of children with $\mathrm{AD}$ were more likely to have a personal history of atopy and a higher educational attainment and social class (Table 2). The annual period prevalence of child $\mathrm{AD}$ ranged from $16.4 \%$ to $21.0 \%$, and $21.8 \%$ to $38.5 \%$ of children with AD were reported to have moderate to severe disease at any given time point. Overall, 4767 children of 13988 (34.1\%) met the definition of having AD between 2 and 11 years of age.

The proportion of all mothers who reported each of the 5 sleep outcomes was fairly consistent across ages: $5 \%$ to $12 \%$ reported sleeping less than 6 hours per night, 18\% to $20 \%$ reported early morning awakenings, $12 \%$ to $13 \%$ reported difficulty falling asleep, $38 \%$ to $43 \%$ reported subjectively 


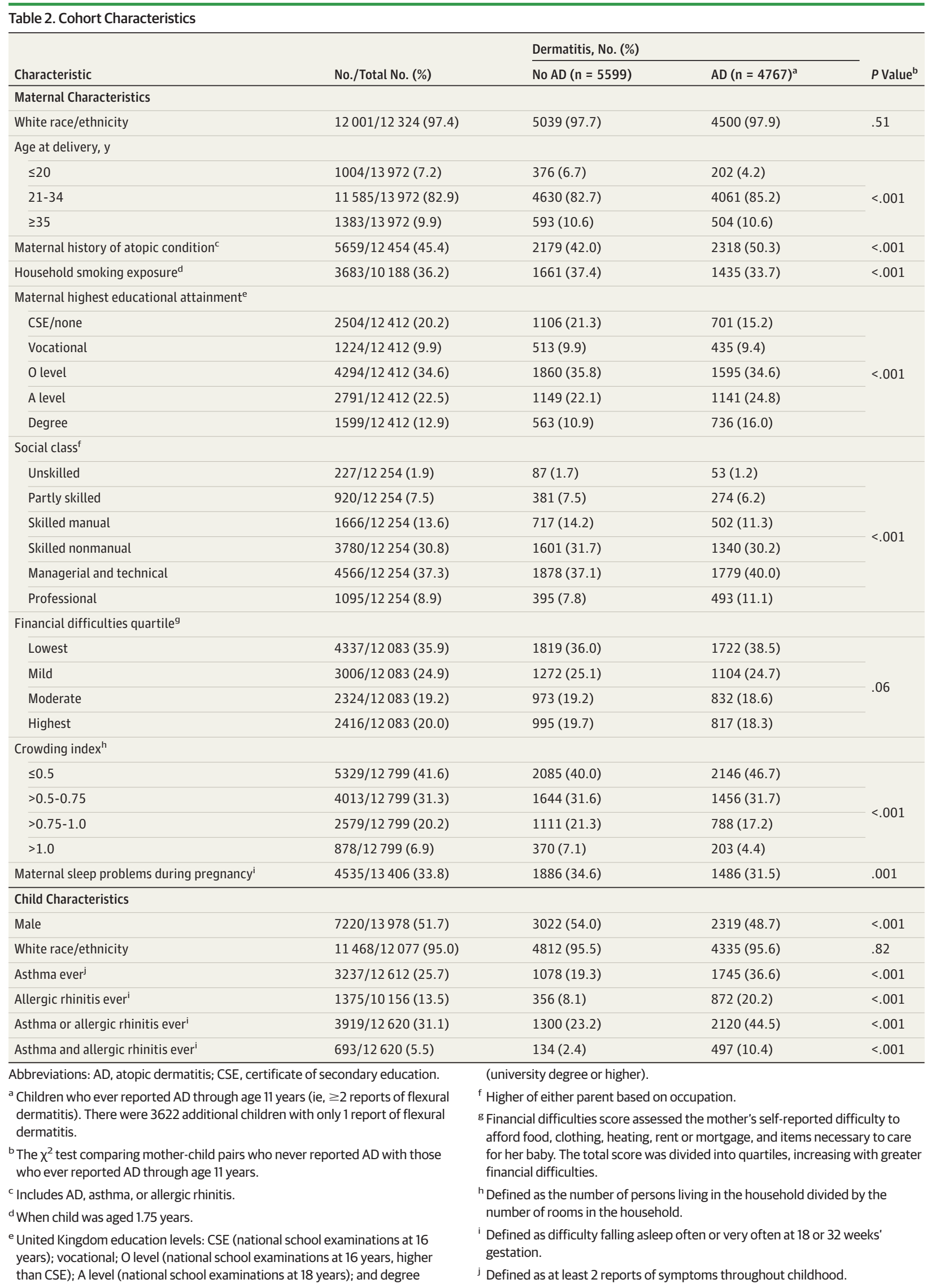


Table 3. Odds of Maternal Sleep Disturbances Across Time Points Among Mothers of Children With Active Atopic Dermatitis (AD) Compared With Mothers of Children Who Never Reported $A D^{a}$

\begin{tabular}{|c|c|c|c|c|c|}
\hline \multirow[b]{2}{*}{ Model } & \multicolumn{5}{|l|}{ Odds Ratio $(95 \% \mathrm{Cl})$} \\
\hline & $\begin{array}{l}\text { Sleep Duration <6 h per } \\
\text { Night }^{\mathrm{b}}\end{array}$ & Early Morning Awakening ${ }^{c}$ & Difficulty Falling Asleep ${ }^{d}$ & $\begin{array}{l}\text { Subjectively Insufficient } \\
\text { Sleep }^{\mathrm{e}}\end{array}$ & Daytime Exhaustion $^{f}$ \\
\hline Unadjusted & $0.92(0.78-1.09)$ & $1.27(1.06-1.52)$ & $1.17(0.96-1.43)$ & $1.45(1.27-1.66)$ & $1.38(1.13-1.69)$ \\
\hline Model 1 & $1.09(0.90-1.32)$ & $1.16(0.93-1.46)$ & $1.36(1.01-1.83)$ & $1.43(1.24-1.66)$ & $1.41(1.12-1.78)$ \\
\hline Model 2 & $1.06(0.85-1.33)$ & $1.15(0.91-1.44)$ & $1.33(0.99-1.79)$ & $1.42(1.23-1.65)$ & $1.43(1.12-1.83)$ \\
\hline
\end{tabular}

${ }^{a}$ For each of the maternal sleep outcomes, results from an unadjusted and 2 separate adjusted multivariable mixed models examining the association between child active $A D$ and maternal sleep disturbances at multiple time points are shown. Model 1 adjusted for child sex, child age, mother's race/ethnicity, child atopy (asthma and/or allergic rhinitis), household smoking exposure, maternal educational attainment, social class, crowding index, financial difficulties score, maternal sleep problems during pregnancy, maternal atopy, and maternal age at delivery. Model 2 adjusted for the same variables as model 1 as well as child sleep disturbances at each time point. Model 2 excluded the 134-month time point from analyses for the outcomes of sleep duration and daytime exhaustion because data on child sleep disturbances were not available at that time.

b Unadjusted model: 10691 individuals; mean (range) observations per individual, 3.7 (1-5). Model 1: 8961 individuals; mean (range) observations per individual, 3.7(1-5). Model 2: 8837 individuals; mean (range) observations per individual, 3.1 (1-4).

c Unadjusted model: 10449 individuals; mean (range) observations per individual, 2.5 (1-3). Model 1: 8796 individuals; mean (range) observations per individual, 2.5 (1-3). Model 2: 8771 individuals; mean (range) observations per individual, 2.4 (1-3)

dUnadjusted model: 10443 individuals; mean (range) observations per individual, 2.5 (1-3). Model 1: 8794 individuals; mean (range) observations per individual, 2.5 (1-3). Model 2: 8768 individuals; mean (range) observations per individual, 2.4 (1-3).

e Unadjusted model: 10546 individuals; mean (range) observations per individual, 3.1 (1-4). Model 1: 8883 individuals; mean (range) observations per individual, 3.1 (1-4). Model 2: 8831 individuals; mean (range) observations per individual, $3.0(1-4)$.

${ }^{f}$ Unadjusted model: 10886 individuals; mean (range) observations per individual, 5.1 (1-7). Model 1: 9061 individuals; mean (range) observations per individual, 5.0 (1-7). Model 2: 9003 individuals; mean (range) observations per individual, 4.4 (1-6). insufficient sleep, and 6\% to $10 \%$ reported daytime exhaustion during the first 11 years of their child's life.

In unadjusted cross-sectional analyses comparing mothers of children with and without $\mathrm{AD}$ at individual time points, results were generally consistent across all child ages (eTable 1 in the Supplement). In longitudinal analyses adjusted for confounders, sleep duration (adjusted OR [AOR], 1.09; 95\% CI, 0.90-1.32) and early morning awakening (AOR, 1.16; 95\% CI, 0.93-1.46) were similar for mothers of children with active $A D$ compared with mothers of children who never reported $\mathrm{AD}$ (Table 3). In contrast, mothers of children with active AD were more likely to report difficulty falling asleep (AOR, 1.36; 95\% CI, 1.01-1.83), subjectively insufficient sleep (AOR, 1.43; 95\% CI, 1.24-1.66), and daytime exhaustion (AOR, 1.41; 95\% CI, 1.121.78) (Table 3). None of the interactions between child AD and child comorbid atopic disease (asthma or allergic rhinitis), child age, child sex, and maternal educational attainment were statistically significant.

\section{Subanalysis With Child AD Disease Severity}

When we examined the odds of maternal sleep disturbances according to the child's disease severity, we found larger effects among mothers of children with more severe disease (Figure and eTable 2 in the Supplement). For maternal sleep duration, we only found a statistically significant association for mothers of children with severe disease, who had $61 \%$ greater odds of reporting sleep duration less than 6 hours per night (AOR, 1.61; 95\% CI, 1.05-2.48). Although the odds were also greater for early morning awakening and difficulty falling asleep, these odds did not reach statistical significance (early awakening: AOR, 1.53; 95\% CI, 0.96-2.45; difficulty falling asleep: AOR, 1.53; 95\% CI, 0.90-2.58). An association with daytime exhaustion and subjectively insufficient sleep was stronger for mothers of children with severe disease
(72\%-89\% greater odds; subjectively insufficient sleep, OR, 1.89; 95\% CI, 1.34-2.66; daytime exhaustion, OR, 1.72; 95\% CI, $1.12-2.64)$ and remained significant for those with mild to moderate disease (31\%-39\% greater odds; moderate disease: subjectively insufficient sleep, OR, 1.31; 95\% CI, 1.09-1.57; daytime exhaustion, OR, 1.38; 95\% CI, 1.06-1.81; mild disease: subjectively insufficient sleep, OR, 1.34; 95\% CI, 1.171.54; daytime exhaustion, OR, 1.39; 95\% CI, 1.12-1.74).

\section{Subanalysis Accounting for Child Sleep Disturbances}

Finally, we recalculated the odds of maternal sleep disturbances while controlling for child sleep disturbances at each time point to examine whether child sleep accounted for most of the association between child $\mathrm{AD}$ and maternal sleep. Overall, we found that the magnitude and significance of the associations was largely unchanged after adjusting for child sleep disturbances in model 2 (Table 3, Figure, and eTable 2 in the Supplement).

\section{Multiple Imputation Results}

Imputed data sets were used to repeat all primary analyses (eTables 2 and 3 in the Supplement). Estimates using the imputed data were slightly attenuated toward the null; however, results remained qualitatively similar and largely consistent with those from the primary data.

\section{Discussion}

In a cohort of 11649 mother-child pairs followed up from the child's birth through age 11 years, sleep duration was similar between mothers of children with and without AD. However, mothers of children with active AD reported subjectively insufficient sleep, difficulty falling asleep, and daytime 
Figure. Adjusted Odds for Sleep Disturbances Across Time Points Among Mothers of Children With Active Atopic Dermatitis (AD) by Disease Severity vs Mothers of Children Who Never Reported AD

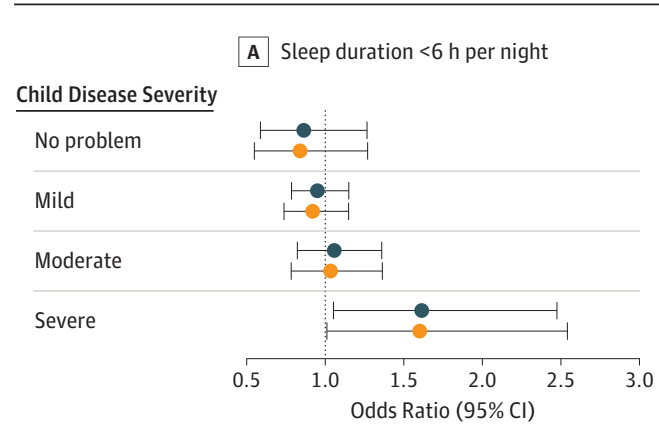

D Subjectively insufficient sleep

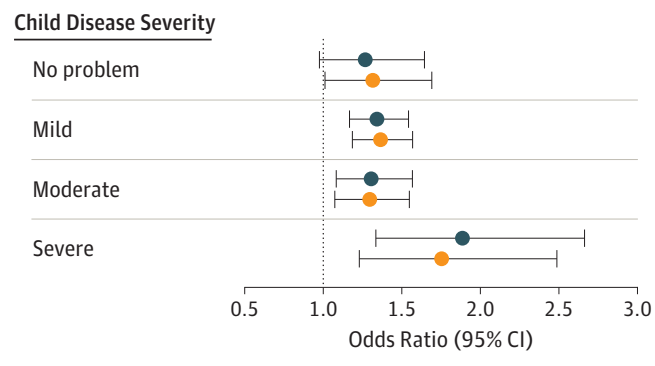

For each of the 5 maternal sleep outcomes, results from 2 separate, adjusted, multivariable mixed models examining the association between child active AD and maternal sleep disturbances at multiple time points are shown. Model 1 is adjusted for child sex, child age, mother's race/ethnicity, child atopy (asthma and/or allergic rhinitis), household smoking exposure, maternal educational attainment, social class, crowding index, financial difficulties score, maternal
B Early morning awakening

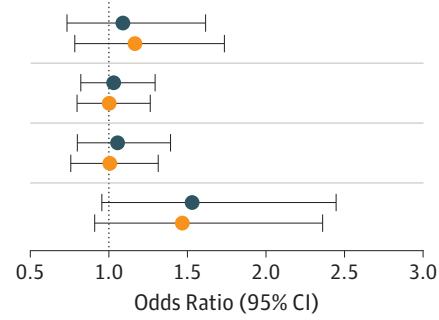

E Daytime exhaustion
C Difficulty falling asleep

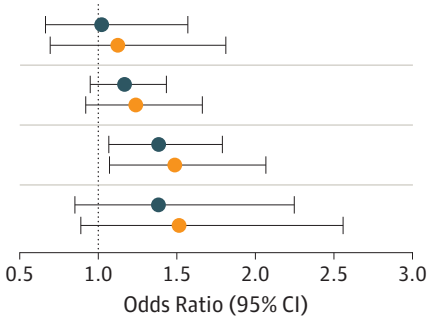

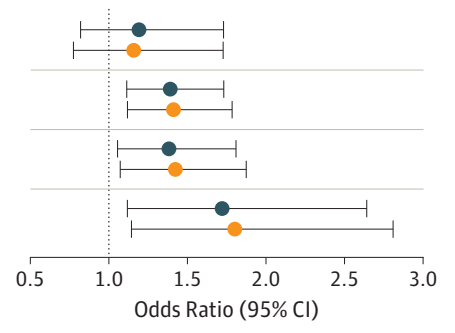

sleep problems during pregnancy, maternal atopy, and maternal age at delivery. Model 2 is adjusted for the same variables as model 1 as well as child sleep disturbances at each time point. Model 2 excluded the 134-month time point from analyses of the outcomes of sleep duration and daytime exhaustion because data on child sleep disturbances were not available at that time.

exhaustion throughout the first 11 years of childhood. These findings have important implications given that $\mathrm{AD}$ is one of the most common chronic childhood conditions and affects up to $20 \%$ of children of any age in industrialized countries. ${ }^{25}$

A strength of the present study is the use of a large prospective, longitudinal, population-based cohort in which children were followed up from birth for more than 10 years. The few studies that have specifically examined the association of child AD with parental sleep relative to parents of children without AD have been small, cross-sectional, and conducted in clinic-based populations. ${ }^{12-15,26}$ In our study, only mothers of children with severe disease were significantly more likely to report sleeping less than 6 hours per night. Similarly, Moore and colleagues ${ }^{26}$ found that parents of children with moderate to severe $\mathrm{AD}$ lost a median of 39 minutes of sleep per night, and Meltzer and Booster ${ }^{13}$ found that parents of children with $\mathrm{AD}$ were significantly more likely to report sleep duration of less than 6 hours per night compared with parents of healthy children.

Although impaired parental sleep is thought to be related to the child's nighttime awakenings, ${ }^{12,13,26}$ adjusting for child sleep disturbances in our study did not change our conclusions. This finding suggests that maternal sleep disturbances may be explained by other factors unrelated to the child's sleep disturbances. Recent work from the pediatric psychology literature in the setting of other chronic illnesses, including asthma, ventilator dependency, and cystic fibrosis, has supported caregiver stress and anxiety related to the child's condition as potential contributors to parental sleep disruptions. ${ }^{7,8,13,27,28}$ Other studies have found that parents of children with AD experience high rates of psychological distress and depression, ${ }^{12,29-31}$ which may explain some of their sleep disturbances. Further research is needed to better understand the exact causes of these sleep disruptions and to characterize their consequences on daytime functioning, well-being, and health outcomes in caregivers of children with $\mathrm{AD}$.

Maternal emotional and psychosocial well-being is inextricably linked to the child's health, development, and cognitive and social functioning. ${ }^{1,2,32}$ In children with $\mathrm{AD}$, chronically sleep-deprived, exhausted, or depressed caregivers may be less equipped to implement time-consuming treatment regimens, regulate their child's behavior, and help the child cope with his or her illness. ${ }^{13,33}$ The American Academy of Pediatrics recommends that clinicians provide family-oriented care that addresses the needs of the entire family and promotes family functioning to optimize child outcomes. ${ }^{1}$ These recommendations include screening for family circumstances that may be associated with unfavorable outcomes for the well-being of the child. Our results suggest that clinicians caring for children with AD should screen for caregiver sleep disturbances and fatigue, engage mothers in discussion about their emotional health, and consider offering resources for psychoso- 
cial support, particularly for mothers of children with more severe disease. Clinicians may also consider formally screening mothers for sleep problems using the Pittsburgh Sleep Quality Index, ${ }^{34}$ for depression using the Patient Health Questionnaire-9, ${ }^{35,36}$ and for anxiety using the Generalized Anxiety Disorder 7-item scale, ${ }^{37}$ all of which are wellvalidated questionnaires. Additional research is needed to understand how interventions targeted to both child AD and maternal wellness can improve the entire family's sleep.

\section{Limitations}

Several limitations warrant discussion, including the potential for selection bias due to missing data and attrition, which is inherent to all large-scale longitudinal studies. To address this limitation, we performed multiple imputations and, reassuringly, found similar results. Another important limitation relates to the possibility for misclassification bias because both exposure and outcome data were self-reported. Although we cannot rule out the possibility of some misclassification, previous studies have found that self-report of $\mathrm{AD}$ closely approximates physician assessment. ${ }^{38}$ Although it is possible that mothers who are chronically exhausted may have a tendency to report more severe disease in their child, the associations remained significant for mothers of children with mild disease. In addition, the estimates of moderate to severe disease match those of other population-based studies, ${ }^{24,39}$ and parent-reported disease severity has been shown to correlate well with objective disease severity measures. ${ }^{40,41}$ In population-based studies comparing sur- vey responses about sleep duration with objective measures, self-report tends to overestimate total duration of sleep, ${ }^{42}$ which would bias our results toward the null. An additional limitation is that the sleep quality questions about difficulty falling asleep and early morning awakenings were not asked beyond the child's fifth year. Future work should investigate whether these maternal sleep quality disturbances persist into later childhood and adolescence. The present study focused on mothers' sleep because mothers are often the primary caregivers; future research should consider the role of fathers and other caregivers. Finally, although the cohort is fairly representative of the United Kingdom's population, ${ }^{16}$ the extent to which our results are generalizable to other populations is unclear.

\section{Conclusions}

This study's results found that, throughout the first 11 years of childhood, mothers of children with active AD experienced more difficulty falling asleep, subjectively insufficient sleep, and daytime exhaustion compared with mothers of children without $\mathrm{AD}$, and the child's disease severity was associated with worse maternal sleep. Child sleep disturbances did not fully explain maternal sleep disturbances, and further research is needed to better understand these mechanisms. In caring for children with $\mathrm{AD}$, clinicians should inquire about caregiver sleep disturbances and fatigue and consider offering psychosocial support.

\section{ARTICLE INFORMATION}

Accepted for Publication: December 10, 2019.

Published Online: March 20, 2019.

doi:10.1001/jamadermatol.2018.5641

Open Access: This is an open access article distributed under the terms of the CC-BY License. (C) 2019 Ramirez FD et al. JAMA Dermatology.

Author Affiliations: Medical student, Department of Dermatology Program for Clinical Research, University of California, San Francisco (Ramirez, Chen); Faculty of Epidemiology and Population Health, London School of Hygiene and Tropical Medicine, London, United Kingdom (Langan); Department of Psychiatry, University of California, San Francisco (Prather); Department of Dermatology Program for Clinical Research, University of California, San Francisco (McCulloch, Kidd, Abuabara); Department of Epidemiology \& Biostatistics, University of California, San Francisco (Cabana); Department of Pediatrics, University of California, San Francisco (Cabana); Philip R. Lee Institute for Health Policy Studies, University of California, San Francisco (Cabana); Department of Dermatology, Vanderbilt University Medical Center, Nashville, Tennessee (Chren).

Author Contributions: Ms Ramirez and Dr Abuabara had full access to all the data in the study and take responsibility for the integrity of the data and the accuracy of the data analysis. Concept and design: Ramirez, Abuabara. Acquisition, analysis, or interpretation of data: All authors.

Drafting of the manuscript: Ramirez, Prather,

\section{Cabana, Abuabara.}

Critical revision of the manuscript for important intellectual content: Chen, Langan, Prather, McCulloch, Kidd, Cabana, Chren, Abuabara. Statistical analysis: Ramirez, Chen, McCulloch, Kidd, Abuabara.

Obtained funding: Abuabara.

Administrative, technical, or material support: Prather, Cabana, Abuabara.

Supervision: Langan, Prather, Abuabara.

Conflict of Interest Disclosures: Ms Ramirez and Dr McCulloch reported receiving grants from the National Institutes of Health. Dr Langan reported receiving grants from Wellcome Trust. Dr Abuabara reported receiving funding for atopic dermatitis research from the National Eczema Association, Dermatology Foundation, Robert Wood Johnson Foundation, and National Institute of Arthritis and Musculoskeletal and Skin Diseases and being a consultant to TARGET Pharma, a company developing a prospective atopic dermatitis registry. No other disclosures were reported.

Funding/Support: The UK Medical Research Council and Wellcome (grant 102215/2/13/2) and the University of Bristol provide core support for the Avon Longitudinal Study of Parents and Children (ALSPAC). This publication is the work of the authors who will serve as guarantors for the contents of this paper. A comprehensive list of grants funding is available on the ALSPAC website (http://www.bristol.ac.uk/alspac/external/ documents/grant-acknowledgements.pdf). This research was specifically funded by the National Eczema Association (Dr Abuabara); the National
Center for Advancing Translational Sciences, National Institutes of Health (NIH); and grant TL1TR001871 received through the University of California, San Francisco Clinical \& Translational Science Institute (Ms Ramirez). Dr Langan is supported by Wellcome Senior Clinical Fellowship in Science (grant 205039/Z/16/Z). Dr Abuabara is supported by grants from the Dermatology Foundation and the Robert Wood Johnson Foundation. Drs Abuabara and McCulloch are supported by grant KL2TR001870 from the NIH.

Role of the Funder/Sponsor: The funders had no role in the design and conduct of the study; collection, management, analysis, and interpretation of the data; preparation, review, or approval of the manuscript; and decision to submit the manuscript for publication.

Disclaimer: The contents of this article are solely the responsibility of the authors and do not represent the official views of the funders.

Additional Contributions: Torsten Neilands, PhD, University of California, San Francisco, provided guidance on the imputation process and was compensated for his work on this manuscript. The authors thank all the families who took part in this study, the midwives for their help in recruiting the families, and the entire ALSPAC team, which includes interviewers, computer and laboratory technicians, clerical workers, research scientists, volunteers, managers, receptionists, and nurses. 


\section{REFERENCES}

1. Schor EL; American Academy of Pediatrics Task Force on the Family. Family pediatrics: report of the Task Force on the Family. Pediatrics. 2003;111(6, pt 2):1541-1571

2. National Research Council, Institute of Medicine. Children's Health, The Nation's Wealth: Assessing and Improving Child Health. Washington, DC: National Academies Press; 2004.

3. Colten HR, Altevogt BM, eds. Sleep Disorders and Sleep Deprivation: An Unmet Public Health Problem. Washington, DC: National Academies Press 2006.

4. Prather AA, Leung CW. Association of insufficient sleep with respiratory infection among adults in the United States. JAMA Intern Med. 2016 176(6):850-852. doi:10.1001/jamainternmed.2016. 0787

5. Markwald RR, Melanson EL, Smith MR, et al. Impact of insufficient sleep on total daily energy expenditure, food intake, and weight gain. Proc Natl Acad Sci U S A. 2013;110(14):5695-5700. doi:10 1073/pnas.1216951110

6. Cappuccio FP, D'Elia L, Strazzullo P, Miller MA. Sleep duration and all-cause mortality: a systematic review and meta-analysis of prospective studies. Sleep. 2010;33(5):585-592. doi:10.1093/sleep/33.5 585

7. Meltzer LJ, Mindell JA. Impact of a child's chronic illness on maternal sleep and daytime functioning. Arch Intern Med. 2006;166(16):1749-1755. doi:10. 1001/archinte.166.16.1749

8. Meltzer LJ, Moore M. Sleep disruptions in parents of children and adolescents with chronic illnesses: prevalence, causes, and consequences. J Pediatr Psychol. 2008;33(3):279-291. doi:10. 1093/jpepsy/jsm118

9. Weidinger S, Novak N. Atopic dermatitis. Lancet 2016;387(10023):1109-1122. doi:10.1016/S0140-6736 (15)00149-X

10. Fishbein $A B$, Vitaterna $O$, Haugh IM, et al Nocturnal eczema: review of sleep and circadian rhythms in children with atopic dermatitis and future research directions. J Allergy Clin Immunol. 2015;136(5):1170-1177. doi:10.1016/j.jaci.2015.08.028 11. Sack R, Hanifin J. Scratching below the surface of sleep and itch. Sleep Med Rev. 2010;14(6):349-350. doi:10.1016/j.smrv.2010.03.003

12. Lawson V, Lewis-Jones MS, Finlay AY, Reid $P$, Owens RG. The family impact of childhood atopic dermatitis: the Dermatitis Family Impact Questionnaire. Br J Dermatol. 1998;138(1):107-113. doi:10.1046/j.1365-2133.1998.02034.x

13. Meltzer LJ, Booster GD. Sleep disturbance in caregivers of children with respiratory and atopic disease. J Pediatr Psychol. 2016;41(6):643-650. doi: 10.1093/jpepsy/jsw016

14. Reid P, Lewis-Jones MS. Sleep difficulties and their management in preschoolers with atopic eczema. Clin Exp Dermatol. 1995;20(1):38-41. doi: 10.1111/j.1365-2230.1995.tb01280.x

15. Chamlin SL, Mattson CL, Frieden IJ, et al. The price of pruritus: sleep disturbance and cosleeping in atopic dermatitis. Arch Pediatr Adolesc Med. 2005;159(8):745-750. doi:10.1001/archpedi.159.8. 745
16. Boyd A, Golding J, Macleod J, et al. Cohort profile: the "children of the $90 \mathrm{~s}^{-}$-the index offspring of the Avon Longitudinal Study of Parents and Children. Int J Epidemiol. 2013;42(1):111-127. doi: 10.1093/ije/dys064

17. Fraser $A$, Macdonald-Wallis $C$, Tilling $K$, et al. Cohort profile: the Avon Longitudinal Study of Parents and Children: ALSPAC mothers cohort. Int J Epidemiol. 2013;42(1):97-110. doi:10.1093/ije/dys066

18. Avon Longitudinal Study of Parents and Children: explore data and samples. University of Bristol website. http://www.bristol.ac.uk/alspac/ researchers/our-data/. Accessed September 1 , 2018.

19. Asher MI, Keil U, Anderson HR, et al. International Study of Asthma and Allergies in Childhood (ISAAC): rationale and methods. Eur Respir J. 1995;8(3):483-491. doi:10.1183/09031936. 95.08030483

20. Perkin MR, Strachan DP, Williams HC, Kennedy CT, Golding J; ALSPAC Study Team. Natural history of atopic dermatitis and its relationship to serum total immunoglobulin $\mathrm{E}$ in a population-based birth cohort study. Pediatr Allergy Immunol. 2004;15(3): 221-229. doi:10.1111/j.1399-3038.2004.00160.x

21. Wadonda-Kabondo N, Sterne JA, Golding J, Kennedy CT, Archer CB, Dunnill MG; ALSPAC Study Team. A prospective study of the prevalence and incidence of atopic dermatitis in children aged 0-42 months. Br J Dermatol. 2003;149(5):1023-1028. doi:10.1111/j.1365-2133.2003.05605.x

22. Henderson J, Northstone $K$, Lee SP, et al. The burden of disease associated with filaggrin mutations: a population-based, longitudinal birth cohort study. J Allergy Clin Immunol. 2008;121(4): 872-877.e9. doi:10.1016/j.jaci.2008.01.026

23. Hirshkowitz M, Whiton K, Albert SM, et al. National Sleep Foundation's updated sleep duration recommendations: final report. Sleep Health. 2015 1(4):233-243. doi:10.1016/j.sleh.2015.10.004

24. Ramirez FD, Chen $S$, Langan $S M$, et al. Association of atopic dermatitis with sleep quality in children [published online March 4, 2019]. JAMA Pediatr. doi:10.1001/jamapediatrics.2019.0025

25. Odhiambo JA, Williams HC, Clayton TO, Robertson CF, Asher MI; ISAAC Phase Three Study Group. Global variations in prevalence of eczema symptoms in children from ISAAC Phase Three. J Allergy Clin Immunol. 2009;124(6):1251-1258.e23. doi:10.1016/j.jaci.2009.10.009

26. Moore K, David TJ, Murray CS, Child F Arkwright PD. Effect of childhood eczema and asthma on parental sleep and well-being: a prospective comparative study. Br J Dermatol. 2006;154(3):514-518. doi:10.1111/j.1365-2133.2005 $07082 x$

27. Meltzer LJ, Pugliese CE. Sleep in young children with asthma and their parents. J Child Health Care. 2017;21(3):301-311. doi:10.1177/1367493517712064

28. Johnson DA, Meltzer LJ, Zhang T, et al. The influence of psychosocial stressors and socioeconomic status on sleep among caregivers of teenagers with asthma, the Puff City Study. Sleep Health. 2018;4(2):141-146. doi:10.1016/j.sleh.2017.11. 003

29. Beattie PE, Lewis-Jones MS. An audit of the impact of a consultation with a paediatric dermatology team on quality of life in infants with atopic eczema and their families: further validation of the Infants' Dermatitis Quality of Life Index and Dermatitis Family Impact score. Br J Dermatol. 2006;155(6):1249-1255. doi:10.1111/j.1365-2133.2006. 07525.x

30. Warschburger P, Buchholz HT, Petermann F. Psychological adjustment in parents of young children with atopic dermatitis: which factors predict parental quality of life? Br J Dermatol. 2004;150(2):304-311. doi:10.1111/j.1365-2133.2004 05743.x

31. Daud LR, Garralda ME, David TJ. Psychosocial adjustment in preschool children with atopic eczema. Arch Dis Child. 1993;69(6):670-676. doi 10.1136/adc.69.6.670

32. England MJ, Sim LJ, eds. Depression in Parents, Parenting, and Children: Opportunities to Improve Identification, Treatment, and Prevention. Washington, DC: National Academies Press; 2009.

33. Teyhan A, Galobardes B, Henderson J. Child allergic symptoms and mental well-being: the role of maternal anxiety and depression. J Pediatr. 2014; 165(3):592-599.e5. doi:10.1016/j.jpeds.2014.05.023

34. Mollayeva T, Thurairajah $P$, Burton $K$, Mollayeva S, Shapiro CM, Colantonio A. The Pittsburgh Sleep Quality Index as a screening tool for sleep dysfunction in clinical and non-clinical samples: a systematic review and meta-analysis. Sleep Med Rev. 2016;25:52-73. doi:10.1016/j.smrv.2015.01.009 35. Spitzer RL, Kroenke K, Williams JB. Validation and utility of a self-report version of PRIME-MD: the PHQ primary care study. JAMA. 1999;282(18):17371744. doi:10.1001/jama.282.18.1737

36. Kroenke K, Spitzer RL, Williams JB. The PHQ-9: validity of a brief depression severity measure. J Gen Intern Med. 2001;16(9):606-613. doi:10. 1046/j.1525-1497.2001.016009606.x

37. Spitzer RL, Kroenke K, Williams JB, Löwe B. A brief measure for assessing generalized anxiety disorder: the GAD-7. Arch Intern Med. 2006;166 (10):1092-1097. doi:10.1001/archinte.166.10.1092

38. Silverberg JI, Patel $\mathrm{N}$, Immaneni $\mathrm{S}$, et al. Assessment of atopic dermatitis using self-report and caregiver report: a multicentre validation study. Br J Dermatol. 2015;173(6):1400-1404. doi:10.1111/ bjd. 14031

39. Silverberg Jl, Simpson EL. Associations of childhood eczema severity: a US population-based study. Dermatitis. 2014;25(3):107-114. doi:10 1097/DER.0000000000000034

40. Vakharia PP, Chopra R, Sacotte R, et al. Validation of patient-reported global severity of atopic dermatitis in adults. Allergy. 2018;73(2):451458. doi:10.1111/all.13309

41. Chang J, Bilker WB, Hoffstad O, Margolis DJ. Cross-sectional comparisons of patient-reported disease control, disease severity and symptom frequency in children with atopic dermatitis. $\mathrm{Br} J$ Dermatol. 2017;177(4):e114-e115. doi:10.1111/bjd.15403

42. Kurina LM, McClintock MK, Chen J-H, Waite LJ, Thisted RA, Lauderdale DS. Sleep duration and all-cause mortality: a critical review of measurement and associations. Ann Epidemiol. 2013;23(6):361-370. doi:10.1016/j.annepidem.2013. 03.015 